\title{
METABOLISM
}

\section{Lathosterone for longer life}

PLoS Biol., published online 10 April 2012 doi:10.1371/journal.pbio.1001305

In Caenorhabditis elegans, bile acid-like steroids called dafachronic acids (DAs) serve as ligands for the nuclear hormone receptor DAF12, which regulates developmental progression and lifespan. DAs are synthesized from dietary cholesterol in a pathway for which several key biosynthetic enzymes and intermediates remain unknown. Now, Wollam et al. apply a genome-wide RNAi screen to identify DHS-16, a conserved short-chain dehydrogenase/ reductase, as a new component of the DA biosynthetic pathway. The phenotype of $d h s-16$ mutants is characteristic of DA deficiency-gonadal migration defects and constitutive dauer formation. Lipid extracts from dhs-16 mutants had less lathosterone and $\Delta^{7}$-DA, its downstream product, than extracts from wild-type animals. Supplementation of the mutants with sterol intermediates pointed to a specific role for DHS-16 in the production of the 3-ketosteroid lathosterone from 3-alcohol sterols such as lathosterol. The authors provided further support for the enzymatic activity of DHS-16 by expressing the protein in HEK293 cells and detecting lathosterone in lipid extracts. It is established that germline-deficient worms live longer and that DAs mediate the lifespan extension. The authors showed that DHS-16 also functions in the gonadal longevity pathway, as it was necessary for the long lifespan of germline-less worms. Future studies will show whether similar small-molecule metabolites and the enzymes controlling their biosynthesis can influence mammalian lifespan.

\section{BIOSYNTHESIS}

\section{Malonyl? Stet.}

Chem. Biol. 19, 329-339 (2012)

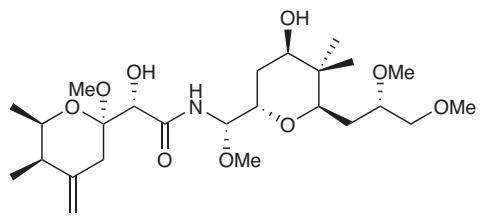

Natural product biosynthesis machineries use a combination of enzyme modules to create a diverse array of natural products. Within polyketide synthases, acyltransferases (ATs) are responsible for loading substrates onto appropriate carrier proteins, with some pathways containing both a 'standard' AT (AT1) and what seems to be a duplicate copy of the AT domain (AT2). A previous observation of premature release of pathway intermediates in a mutant of the bacillaene cluster suggested the pathway contained a proofreading mechanism, but the protein responsible for this function was unknown. In efforts to track down this enzyme, Jensen et al. noted that ATs are functionally related to the putative proofreader. To explore this idea, they focused on the pederin pathway, which contains one AT1 (PedD) and one AT2 (PedC). In vitro tests of both enzymes showed that, in line with normal AT function, PedD could transfer malonyl units onto any of four carrier proteins. PedC, however, was inactive. In contrast, assays testing for possible proofreading activity that showed PedD was inactive, whereas PedC was able to hydrolyze four of six putative substrates. PedC was further tested on carrier proteins modified with hexanoyl, malonyl or acetyl groups and hydrolyzed the hexanoyl and acetyl but not malonyl groups. These combined results indicate that PedC, and most likely other AT2 homologs, determine product integrity by allowing incorporation of only the correct malonyl substrates. $C G$

CHEMICAL PROBES

Motor control

Nature 484, 125-129 (2012)

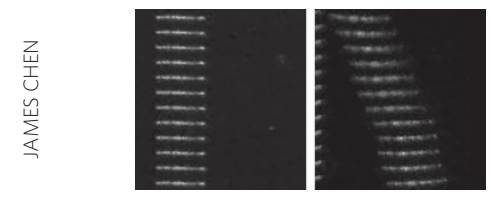

Cytoplasmic dynein is a minus end-directed molecular motor in the AAA+ family of ATPases that couples ATP hydrolysis to the generation of mechanical force. In an effort to identify new cellular targets in the Hedgehog $(\mathrm{Hh})$ pathway, Firestone et al. focused on ciliobrevins, also known as 2,4-dichlorobenzoyl dihydroquinazolinones, which were identified in a previously published high-throughput chemical screen for inhibitors of Hh signaling. Importantly, long-term exposure to the compounds not only blocked Hh signaling but also induced structural defects in the primary cilium, the site for active Hh signaling. Short-term application of ciliobrevins to cells increased amounts of ciliary Gli2, a transcription factor in the Hh pathway; on the basis of this observation, the authors hypothesized and confirmed that the compounds disrupted protein transport from the ciliary tip toward the cell body, which depends on cytoplasmic dynein 2 . The authors further showed that ciliobrevins disrupted cellular processes dependent upon the activity of cytoplasmic dynein 1 , a closely related isoform, including mitotic spindle pole assembly, kinetochore-microtubule attachment and organelle trafficking. In vitro, ciliobrevins dose-dependently inhibited the movement of fluorescence-tagged microtubules on dynein-coated glass slides (see image) and similarly inhibited the ATPase activity of dynein, but not other motor proteins, in an ATP-competitive manner. Collectively, these data support the conclusion that ciliobrevins are the first direct and selective inhibitors of the ATPase cytoplasmic dynein.

CELL BIOLOGY

\section{Peroxisomes come together Cell 149, 397-409 (2012)}

Organelles generally either proliferate from preexisting organelles and then import necessary proteins from the cytosol or are derived in full from the endoplasmic reticulum (ER). Peroxisome biogenesis seems to combine elements of both strategies, with ER-derived membranes and transport proteins responsible for importing cytosolic proteins, but the way in which these pathways work together remains controversial. Now, Van der Zand et al. demonstrate that the combined path is made possible by the separate trafficking of peroxisomal components. The authors used bimolecular fluorescence complementation in combination with mutants at various steps in the biogenesis pathway to observe assembly of proteins from the two halves of the peroxisomal translocon, the docking complex and the RING finger complex. In mutants where ER export or vesicle fusion was blocked, the docking complex proteins assembled together, as did the RING finger complex proteins, but inter-complex assembly was not observed. Biochemical isolation, coimmunoprecipitation and fluorescence experiments on the unfused, immature vesicles indicated that these protein complexes were segregated in separate vesicles; pulse-chase experiments in wild-type cells confirmed this result. The authors further showed that the separate vesicles are on-pathway intermediates; after fusion of two preperoxisomal fractions, translocon assembly allowed import of a fluorescent protein tagged with a peroxisomal targeting signal. These surprising results explain the dual biogenesis strategy and raise new questions regarding protein trafficking in the ER. 